\title{
Redes temáticas em fotologs: proposições iniciais para o estudo de caso sobre Síndrome de Down (SD)
}

Prof. Dra. Sandra Portella Montardo (Feevale). E-mail: sandramontardo@ feevale.br Elias Ferreira Goedtel (BIC-Fapergs/Feevale). E-mail: eliasferreirag@ hotmail.com Maite Etchegaray Scherer (BIC-CNPq/Feevale). E-mail: maitees@ feevale.br

Resumo: A partir do entendimento de que blogs e fotologs podem ser ferramentas de socialização e de inclusão de Pessoas com Necessidades Sociais (PNE), este artigo pretende descrever a netnografia como metodologia para seleção da amostra e obtenção de dados e sua pertinência para o mapeamento de rede de fotologs de pais de crianças com Síndrome de Down (SD). O predomínio absoluto de fotologs nesta rede temática, requer que se examine a relação entre o tema desta rede (SD) e o suporte em que ela se constitui. Para tanto, é necessário investigar o estado da arte sobre fotologs, bem como definições, características e problematizações em torno da SD como subsídios teóricos fundamentais para a definição dos próximos passos da pesquisa.

Palavras-chave: Fotologs; Síndrome de Down; Inclusão Social; Inclusão Digital; Redes Temáticas.

Title: Thematic nets in photologs: initial propositions for a case study about Down's Syndrome.

\begin{abstract}
Starting from the understanding that blogs and photologs may be used as tools for socialization and inclusion of disabled people, this paper intends to describe netnography as methodology to select sample and to obtain data, and its relevance to map the photologs net of parents of Down's Syndrome children. The fact that photologs predominate in this thematic net demands an analysis of the relation between the theme of this net (Down's Syndrome) and the media in which it is made up. For this purpose, it is necessary to investigate the state of art on photologs as well definitions, characteristics and considerations about Down's Syndrome.
\end{abstract}

Key words: Photologs; Down's Syndrome; Social Inclusion; Digital Inclusion; Thematic nets.

\section{Introdução}

Nesta pesquisa, partimos da hipótese de que a socialização on-line pode ser fator de inclusão social de Pessoas com Necessidades Especiais (PNE) e de seus familiares, uma vez que ajuda a promover a autonomia dessas pessoas de maneira geral. Em um estudo anterior (Montardo e Passerino, 2008), mapeamos e analisamos uma rede temática sobre autismo e Síndrome de Asperger em blogs, cujos autores são os pais das crianças. Nele, verificamos diferentes tipos de atuação dos autores de blogs quanto aos conteúdos postados e comentados, bem como quanto à intenção comunicativa de cada um. Assim, podemos identificar entre os promotores, aqueles que apenas usufruem da Inclusão Social em blogs, e os que fazem as duas coisas, a partir dos conteúdos disponibilizados nestes (conteúdo informativo ou de apoio emocional). Percebemos, assim, que os blogs serviam como uma extensão de Associação de Pais sobre o tema em questão, com a troca de informações, dicas sobre tratamento e apoio diante dos desafios 
postos pelo autismo e pela Síndrome de Asperger, questões que ainda não estão definidas no campo científico em sua totalidade.

Frente a isso, foi com surpresa que, na busca do mapeamento sobre redes temáticas em blogs sobre a Síndrome de Down (SD), deparamo-nos com uma prevalência absoluta de fotologs de crianças com SD, criados pelos seus pais. Por que a utilização de fotologs nesta rede temática? O que se faz melhor nesse suporte do que em blogs? O que se faz em blogs que não se torna tão relevante para os pais de crianças com SD? $\mathrm{Na}$ busca por estas respostas a estas questões, investigamos questões relativas a redes sociais em fotologs, características da SD, além de descrevermos aspectos da netnografia como metodologia para obtenção de dados e seleção da amostra desta rede temática.

\section{Redes Sociais em Fotologs}

Fotologs são páginas pessoais para postagem de fotografias, nas quais as pessoas podem comentar as mesmas (Recuero, 2006) ou, de maneira mais informal, "mistura de blogs com álbuns de fotografia" (RIGONI, 2006). Observa-se que na definição de Khalid e Dix (s/d), retirada da Wikipédia $^{1}$, a ferramenta de comentários não é mencionada:

\footnotetext{
Photolog or photoblog is a specific type of weblog that allows on to order digital photos systematically, often in chronological order. For many, the photolog is seen as an alternative to the online photo album, which was introduced earlier and is familiar to most online users. The photolog is a form of social software application that allows people collaborate and connect, that uniting many users through photographs. (KHALID; DIX, s/d)
}

Curiosamente, ao lado de sistemas de publicação que permitem a criação de fotologs, Khalid e Dix (s/d) citam Fotolog. $\mathrm{com}^{2}$, Fotopages ${ }^{3}$ e o Flickr ${ }^{4}$. Frente a isso, cabe concluir que os autores não diferenciam álbuns de fotografias (que não necessariamente apresentam ferramenta de comentários), fotologs (cuja principal diferença com relação aos álbuns é a possibilidade de comentários) e o Flickr, sistema de compartilhamento de fotos que permite, também, comentários até mesmo dentro das fotos (notes), bem como a sua classificação em tags. Assim, identifica-se nesses três diferentes suportes mais de um tipo de interação possível, o que é fundamental para a análise que se pretende nesta investigação.

\footnotetext{
${ }^{1}$ Disponível em http://www.wikipedia.com.

${ }^{2}$ Disponível em http://www.fotologs.com.

${ }^{3}$ Disponível em http://www.fotopages.com.

${ }^{4}$ Disponível em http://www.flickr.com.

V. 6 № 1 , Julho, 2008
} 
No entanto, compartilhamento de fotos on-line e comentários a respeito destas cada vez menos passam a ser privilégio de fotologs. Redes sociais de relacionamento como Friendster $^{5}$, Facebook ${ }^{6}$ e Orkut ${ }^{7}$, por exemplo, recentemente têm disponibilizado um número maior de fotos por páginas pessoais, assim como uma ferramenta de comentários.

Assim como os blogs (Amaral, Montardo, Recuero, 2008), os fotologs têm sido objeto de estudo em várias áreas de conhecimento, a partir de diversas perspectivas e metodologias. Com um estilo ensaístico, Bruno (2005) reflete sobre a reconfiguração da relação público e privado a partir do olhar do outro em weblogs, fotologs e reality shows. Aragão (2006), por exemplo, descreveu e analisou usos lingüísticos em sete diferentes suportes da web, dentre os quais os fotologs. Rigoni (2006) buscou na análise de fotologs flagrar valores e práticas de comunicação entre jovens que poderiam ser balizadores de ações pedagógicas em ambientes colaborativos. Aspectos técnicos do fazer fotográfico, por meio da descrição do percurso histórico da fotografia desde o século XIX até a atualidade, com o advento dos fotologs, é a intenção de Ferreira (2005). Já Teles (2005) se ocupa do novo papel da fotografia na era dos fotologs, quanto à sua circulação e participação em novas práticas comunicacionais. Por sua vez, fotologs como experiência temporal da constituição da memória na contemporaneidade na Internet e a visibilidade da vida privada é a abordagem de Sanz $(2005,2006)$.

Ao qualificar o prazer de observar fotologs como "indulgência seletiva", Khalid e Dix (2006) classificam usuários de sistemas de publicação de fotologs como fotologueiros $^{8}$ (quem disponibiliza fotos suas em fotologs) e fotolurkers ${ }^{9}$ (pessoas que postam poucas ou nenhuma foto em fotologs, restringindo-se a observar esses suportes). Em um estudo posterior, os autores (s/d) acrescentam à categoria desses usuários os visitantes, pessoas que não necessariamente têm fotologs, mas que também não são visitantes "silenciosos". Neste mesmo estudo, os autores acrescentam que "the apllication contains some social-networking aspects that influence social interaction online and local groups" (KHALID; DIX, s/d, p. 5). Fica clara, com isso, a vocação colaborativa e comunitária dos fotologs.

\footnotetext{
${ }^{5}$ Disponível em http://www.friendster.com.

${ }^{6}$ Disponível em http://www.facebook.com.

${ }^{7}$ Disponível em http://www.orkut.com.

${ }^{8}$ Photologgers

${ }^{9}$ Photolurkers

V. 6 № 1, Julho, 2008
} 
De acordo com esta mesma abordagem, Recuero (2004) problematiza a teoria das redes e sua aplicação às redes sociais na Internet, por meio da análise do Orkut, de blogs e fotologs. Em sua tese de doutorado, a autora (2006) relaciona os conceitos de rede social na internet com o de comunidades virtuais, analisa a organização, a estrutura e a dinâmica de uma rede social no sistema de publicação Fotolog.com, propondo a seguinte tipologia de comunidades virtuais a partir dos padrões observados: 1) comunidades emergentes, caracterizada pela interação social mútua (Primo, 1998, 2003), pela predominância de laços relacionais (Breiger, 1974), capital social diversificado (Bertolini e Bravo, 2004) e pela ocorrência de trocas comunicacionais; 2) comunidades associativas, marcadas pela predominância de interação social reativa (Primo, 1998, 2003), de laços sociais associativos (Breiger, 1974) e, também, pelo aporte de capital social diversificado (Bertolini e Bravo, 2004); e, finalmente, 3) comunidades híbridas, as quais combinam características dos dois tipos anteriormente apresentados. Em um último estudo, Recuero (2008) propõe o entendimento do conceito de fotolog como site de redes sociais, a partir da análise das apropriações interacionais e de capital social na ferramenta de comentários de 18 fotologs.

Já o estudo de Kuhn Jr (2008), propõe-se uma tipologia para os usos do fotográfico em fotologs como dinamizador de interações na internet. Kuhn Jr. (2008) classifica esses usos do fotográfico da seguinte forma: 1) partilhamento de experiências (molduras de documentação e memória cotidiana; molduras de documentação e memória de deslocamentos especiais; molduras de experimentação narcísea), no 2) partilhamento de qualidade dos conteúdos (colagens fotográficas e colecionismos temáticos; efeito publicidade; iconografia midiática) e, finalmente, no 3) compartilhamento do ato fotográfico em si (pictorialismo infográfico; pictorialismo fotográfico).

Por sua vez, McDonald (2007) analisa o que denomina de "conversações visuais" em comunidades virtuais, alegando que os estudos sobre redes sociais na web se voltem mais para o papel do texto do que das imagens nas interações. No entanto, o autor (2007) não detém essa análise a fotologs ou sites de compartilhamento de fotos, o que não impede que se investigue a viabilidade dessa proposta.

Esta investigação se associa a estas três últimas abordagens, no sentido que visa identificar o potencial inclusivo da socialização em fotologs de pais de pessoas com Síndrome de Down e, para tanto, busca entender os motivos da escolha deste suporte V. 6 № 1 , Julho, 2008 


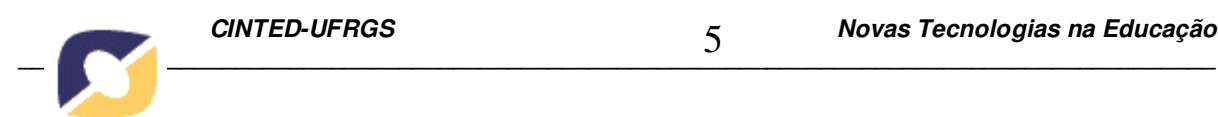

para socialização. Cabe agora, esclarecer características da SD, bem como possíveis problematizações, para o delineamento da compreensão da motivação temática desta rede.

\section{Síndrome de Down (SD)}

De acordo com Bissoto (2005), a SD se caracteriza por uma alteração na divisão cromossômica usual, resultando na triplicação o material genético existente no cromossomo 21, ao invés da duplicação deste.

Segundo Pereira-Silva e Dessen (2002), ainda que os primeiros registros sobre a SD datem do século XIX, obras de pintores como Andrea Mantegna (1431-1506) e Jacob Jordaens (1539-1678) retratam crianças com a síndrome, já que elas são facilmente reconhecidas por traços específicos nos olhos, orelhas e mãos. As autoras (2002) ainda destacam que um dicionário médico traz uma referência de Esquirol sobre a SD em 1838; que Chambers, em 1844, denomina SD como "idiotia do tipo mongolóide", e, finalmente, Edouard Seguin, entre 1846 e 1866, classificava a SD como um "subtipo de

Cretinismo, chamado "cretinismo furfuráceo" (SCHWARTZMAN apud PEREIRA SILVA, DESSEN, 20002). Essas autoras (2002) ainda chamam a atenção que apenas em 1866, com Langdon Down, que a SD aparece como uma manifestação clínica, ainda que apoiada em pressupostos evolucionistas, que creditava a deficiência mental a raças inferiores.

Ainda quanto à evolução dos conhecimento sobre a SD, uma série de estudos quanto às suas causas foram conduzidos até que, em 1932, fosse sugerida algum tipo de aberração cromossômica como causa, por Waardenburg, oftalmologista holandês. Em 1934, Adrian Bleyer supôs a trissomia como ocorrência dessa desordem. E, apenas mais de 20 anos depois, em 1959, é que o Dr. Jerome Lejeune e Patrícia Jacobs descobriram, praticamente ao mesmo tempo, a existência de um cromossomo extra, conforme Schwartzman (1999a) citado por Pereira Silva e Dessen (2002).

Ainda de acordo com Schwartzman (1999), as autoras (2002) mencionam que apesar de SD ter sido conhecida por inúmeras denominações, muitas das quais extremamente pejorativas, nas publicações ligadas à Organização Mundial da Saúde (OMS), predomina a referência de SD desde 1965. 


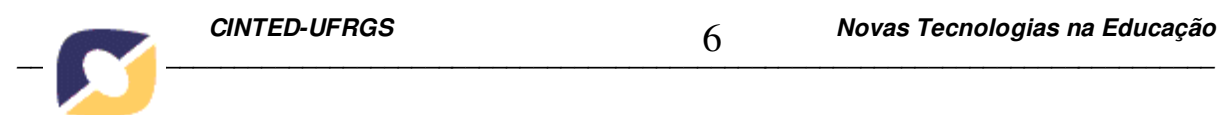

Embora a causa da alteração cromossômica não esteja totalmente esclarecida, Bissoto (2005) aponta que ela pode acontecer de três modos diferentes: a) em 96\% dos casos, a trissomia se apresenta como uma não-disjunção cromossômica total; b) em 0,5 a $1 \%$ dos casos, os portadores não apresentam todas as células afetadas pela trissomia (mosaico); c) há, também, a trissomia causada por translocação gênica, perfazendo de 3 a $3,5 \%$ dos casos.

Para Martini et. al. (2007), a palavra síndrome designa, em medicina, uma “desordem clínica na qual algumas características ou sintomas comumente ocorrem juntos" (MARTINI et. al., 2007, p. 31). No caso da SD, as características são: "cardiopatia congênita (40\%); hipotonia (100\%); problemas de audição (50 a 70\%); de visão (15\% a 50\%); alterações na coluna cervical (1 a 10\%); distúrbios da tireóide (15\%); problemas neurológicos (5 a $0 \%$ ); obesidade e envelhecimento precoce (MOREIRA et. al., 2000, p. 97), fatores estes que acabam por interferir no desenvolvimento global da criança portadora (BISSONI, 2005).

No entanto, ao buscar evidenciar correlações necessárias ao desenvolvimento cognitivo e os processos de aprendizagem da pessoa com SD, Bissoni (2005) questiona uma série de pressupostos teóricos referentes a diagnósticos estáticos da Síndrome, enfatizando a influência dos aspectos sociais, culturais, genéticos e, em decorrência disso, as expectativas que se tem do seu desenvolvimento, bem como aspectos afetivoemocionais aí existentes. Por outras palavras, quanto a isso, o desenvolvimento de um portador de SD se assemelha, em condições, ao de qualquer outra pessoa e, por isso mesmo, cada portador de SD possui um processo de desenvolvimento particular.

Quanto aos fatores da SD, Schwartzman (1999b), citado por Pereira-Silva e Dessen (2002), aponta a idade avançada da mãe, a ausência de diagnóstico pré-natal e a exposição à radiação como fatores relacionados à incidência de SD.

Em um estudo empírico que interrogou pais de crianças com SD sobre o comunicado deste diagnóstico para a criança, Martini et. al. (2007) concluíram que os pais evitam esse comunicado, em parte, por reviver o desamparo sentido com o comunicado de diagnóstico que receberam do médico no momento do nascimento da criança, o qual enfatiza a deficiência e não as possibilidades desta. Ainda que seja possível identificar a SD antes do nascimento, a maioria dos pais toma conhecimento do fato logo após o parto. Ainda que as autoras (2007), através de Manoni (1995) e de Jerusalinski (2002), salientem as diferentes formas de respostas desses pais ao fato de V. 6 № 1 , Julho, 2008 
terem um filho com SD, é comum que ocorra um sentimento de perda, de luto, com relação ao filho ideal imaginado, sendo que, freqüentemente, entendem o bebê com SD como um impostor que tomou o lugar do filho imaginado.

Isso, por sua vez, pode influenciar de maneira significativa a relação pais-filhos, de acordo com as autoras (2007), principalmente no que tange à falta de expectativas dos pais com relação a esses filhos, o que compromete significativamente o seu desenvolvimento como um todo.

Ao refletir sobre o impacto da criança SD na família, Casarin (1999), citado por Silva e Dessen (2002) destacam que "a família precisa repensar o significado da Síndrome de Down para que, reformulando a imagem deformada que possuía, possa constituir uma outra, não idealizada que viabilize seu relacionamento com a pessoa portadora da síndrome (Casarin, 1999 apud PEREIRA SILVA, DESSEN, 2002, p. 170), de forma que o ambiente familiar proporcione o desenvolvimento da criança com SD.

Frente a isso, entende-se que a socialização on-line pode auxiliar no compartilhamento dessas e de outras questões em uma rede temática sobre SD. O próximo íten traz considerações preliminares sobre essa rede ter sido constituída em fotologs.

\section{Procedimentos metodológicos e implicações para a continuidade da pesquisa}

Em artigos anteriores, fez-se considerações sobre o uso da netnografia no estudo de blogs (Montardo, Passerino, 2006) e combinada à Análise de Redes Sociais (ARS) (Montardo, Passerino, 2008).

Em primeiro lugar, é importante destacar que a netnografia é requerida em um primeiro momento da pesquisa, durante os processos de seleção de amostra e de obtenção de dados sobre a rede temática em questão. Quanto a isso, o primeiro processo de levantamento dos blogs sobre SD se deu no site Pesquisa de Blogs Google ${ }^{10}$ e da ferramenta de busca do site Technorati ${ }^{11}$. Em seguida, por meio de comentário nos próprios blogs e fotologs encontrados, explicou-se a pesquisa como um todo, deixou-se um link para o blog da pesquisa (Blogs Especiais) ${ }^{12}$, o qual traz o vínculo dos pesquisadores com a instituição em que se desenvolve o projeto, bem como links para

\footnotetext{
${ }^{10}$ Disponível em http://www.google.com.br.

${ }^{11}$ Disponível em http://www.technorati.com.

${ }^{12}$ Disponível em http://www.blogsespeciais.blogspot.com.

V. 6 № 1, Julho, 2008
} 
os CV de todas as pessoas ligadas ao projeto. Nessa oportunidade, foi solicitada a

\begin{tabular}{|l|l|}
\hline Suporte que constitui rede temática sobre SD & $\begin{array}{l}\text { Número de autorizações de obervação por } \\
\text { número de suporte encontrado }\end{array}$ \\
\hline Fotolog organizado por pais de portadores de SD & 12 autorizações de 37 \\
\hline Fotologs de portadores de Síndrome de Down & 0 autorizações de 1 \\
\hline Blog de pais de portadores de SD & 0 autorizações de 1 \\
\hline Blog de amigos de portadores de SD & 0 autorizações de 2 \\
\hline Fotolog de amigos de portadores de SD & 0 autorizações de 1 \\
\hline Blogs de portadores de Síndrome de Down & 0 autorizações de 2 \\
\hline
\end{tabular}

autorização para observar os blogs e fotologs sobre SD levantados, que deveria ser dada por e-mail, endereçado à líder do projeto. Esse processo foi realizado do início de março a dia 10 de abril de 2008. Segue tabela comparando os blogs e fotologs encontrados, bem como as autorizações recebidas:

Tabela 1. Fotologs e blogs encontrados sobre a Síndrome de Down e autorizações concedidas para a sua observação

Foi sugerido, a partir do Comitê de Ética na Pesquisa (CEP), da Feevale, que se envie a estes autores, um Termo de Compromisso para Uso de Conteúdo dos fotologs encontrados, a fim de firmar o comprometimento de se utilizar os dados somente para fins de pesquisa e sem identificar os fotologs em nenhuma publicação.

Outro passo importante é a transcrição dos comentários e das legendas dos fotologs para tabelas, a fim de que se possa identificar o processo de inclusão social a partir da análise do conteúdo da socialização na rede temática em questão, bem como aspectos referentes à estrutura desta rede. Esse último ponto passa pelo mapeamento das redes, identificando-se quais fotologs estão ligados a quais outros fotologs, quem comenta no fotolog de quem, fotos de qual tipo são mostradas nesses suportes. O mapeamento das redes no software CMap Tools ${ }^{13}$ é fundamental para a visualização desta rede, bem como para as análises pretendidas. Essa segunda etapa da pesquisa sobre redes temáticas sobre SD em fotologs inscreve-se no âmbito da Análise de Redes

\footnotetext{
13 “O CMapTools um software destinado à elaboração de mapas conceituais. Criados pelo psicólogo americano Joseph Novak e baseados na teoria da aprendizagem significativa de David Ausubel. Estes mapas são diagramas bidimensionais com a finalidade de representar conceitos e suas respectivas proposições”. (BENKENSTEIN, MONTARDO, PASSERINO, 2007, p. 7).
}

V. 6 № 1, Julho, 2008 
Sociais (ARS), a partir da qual busca estabelecer padrões de socialização na rede considerada $^{14}$.

$\mathrm{Na}$ medida em que um questionário vai ser elaborado e enviado para os autores de fotologs, cabe que se liste algumas observações preliminares que, juntamente com as questões teóricas levantadas, vão subsidiar sua elaboração. Metodologicamente, cabe destacar que observou-se um aumento de postagens de fotos após o pedido de autorização de observação dos blogs. E, também , que é sempre bem menor o número de fotologs que permitem a autorização dos que os que não permitem.

Quanto a aspectos estruturais da rede, destaca-se que apenas mulheres são autoras dos fotologs de seus filhos e comentadoras dos fotologs da rede. Também destaca-se que os fotologs linkados aos fotologs observados eram predominantemente referentes a crianças com SD. Fala-se em crianças porque foi encontrado apenas um fotolog de adolescente com $\mathrm{SD}$, do qual ele era o seu próprio autor. Da mesma forma foi constatado que havia pouca diversidade entre os comentadores de fotologs.

\section{Considerações finais}

A título de considerações finais, vale destacar que uma hipótese inicial foi redimensionada. A identificação maciça de fotologs sobre SD, combinada à insignificância de ocorrência deste suporte no levantamento e mapeamento de outras redes temáticas deste projeto, instigaram a suposição de que o autismo a Síndrome de Asperger, uma vez que não são imediatamente identificadas nas crianças, levando-se vários anos para tanto, suscita mais debate, o que pode ser facilmente proposto, administrado, localizado em blogs, via postagens e ferramenta de comentários.

Frente a isso, pensou-se inicialmente que não havia muito o que se conversar sobre SD, já que ela é conhecida cientificamente desde o século XIX, sendo detectada até mesmo em exames pré-natal. Some-se a isso, o fato de se trata de uma Síndrome com características fenotípicas facilmente identificáveis (as obras de Mantegna e de Jordaens são uma evidência disso cerca de três séculos antes da definição científica), os fotologs apareceram como uma forma de mostrar a pessoa com SD, o que contraria alguns impulsos ainda existentes, de se evitar de falar sobre filhos com SD, a de se esconder os filhos em casa, e de comunicar a SD para seus portadores (Martini et. al.,

\footnotetext{
${ }^{14}$ Sobre Análise de Redes Sociais aplicada a este projeto, ver Benkenstein, Montardo e Passerino (2007). 
2007). No entanto, deve-se resistir à tendência de se pensar que as fotos evitam conversas, mas podem constituir um tipo de "conversação visual" como propõe McDonald (2007).

Considera-se fundamental essas pistas iniciais para subsidiar a elaboração do questionário para os autores dos blogs, bem como para a aplicação da Análise de Redes Sociais (ARS) para avaliação de ocorrência de inclusão social na rede temática sobre Síndrome de Down. Quanto a isso, é importante ainda destacar que a classificação da amostra de fotologs em uma tipologia (KUHN Jr., 2008) combinada às possibilidades de compreensão da socialização desta rede como conversações visuais em comunidades virtuais (McDonald, 2007) parecem pertinentes para a análise pretendida, no sentido de contemplar os desafios representados pelas diferença de análise de blogs para a de fotologs. Este será o próximo passo desta pesquisa.

\section{Referências:}

AMARAL, A. ; MONTARDO, S. P.; RECUERO, R. Blogs: mapeando um objeto. In: VI Congresso Nacional de História da Mídia. Anais. Niterói, UFF, 2008. Disponível em CD-Rom.

ARAGÃO, R. Descrição e análise dos usos lingüísticos de diferentes ambientes da Internet e sua relação com o suporte. In: Revista Letras. Curitiba. UFPR, no. 70, p. 137-156, Set./Dez. 2006.

BENKNSTEIN, A.;MONTARDO, S. P.; PASSERINO, L. Análise de redes sociais em blogs de Pessoas com Necessidades Especiais (PNE). In: RENOTE (Revista Novas Tecnologias na Educação). Vol. 5, no. 2, Dez. 2007. Disponível em: http://www.cinted.ufrgs.br/renote/dez2007/artigos/6hLilianaPasserino.pdf.

BERTOLINI, S.; BRAVO, G. Social Capital, a Multidimensional Concept.Disponívelemhttp://www.ex.ac.uk/shipss/politics/research/socialcapital/other/bertolini.pdf.

Acesso em 17 out 2004.

BISSOTO, M. L. Desenvolvimento cognitivo e o processo de aprendizagem do portador de Síndrome de Down: revendo concepções e perspectivas educacionais. In: Revista Ciências e Cognição, 2005, Vol. 4, pp. 80-88.

BREIGER, R. The Duality of Persons and Groups. Social Forces, vol 53, n. 2, p.181- 190, dez 1974.

BRUNO, F. Quem está olhando? Variações do público e do privado em weblogs, fotologs e reality shows. In: Revista Contemporânea. Vol. 3, No. 2, 2005.

FERREIRA, G. O percurso da fotografia e seus usos atuais: uma abordagem sobre a imagem digital e foologs como nova mídia. In: XXVIII Intercom. Anais. Rio de Janeiro, UERJ, 2005. Disponível em CdRom.

KHALID, H.; DIX, A. (2007). I know what you did in last Summer.What can we learn from photoblog? Disponível em http://sunschlichter0.informatik.tu-muenchen.de/persons/kochm/ecscw2007ws/paperkhalid.pdf. Acesso em 24 mai. 2008.

From selective indulgence to engagement: exploratory studies of photolurking. The British Human Computer Interaction Conference, 2006. Queen Mary, University of London. Disponível em: http://www.comp.lancs.ac.uk/ dixa/papers/HCI2006-indulgence/khalid-dix-indulgence-final.pdf. Acesso em 23 mai. 2008.

V. 6 № 1 , Julho, 2008 
KHUN Jr., N. Painéis Fotográficos na Internet. Um estudo dos Fotologs como molduras de mostração. Tese de Doutorado. Programa de Pós-Graduação em Ciências da Comunicação. Universidade do Vale do Rio do Sinos. São Leopoldo, 2008.

MARTINI, F. de O.; LIPP, L.K.; SANTOS, T. F. ; OLIVEIRA-MENEGOTTO, L. M. Você tem Síndrome de Down: algumas reflexões sobre a não-comunicação do diagnóstico entre pais e filhos. In: Revista Prâksis. Novo Hamburgo, Ano 4, V. 2, Ago. 2007, pp. 31-37. Disponível em http://www.feevale.br/internas/vwMidia.asp?idGaleria=14\&idMidia=18812\&intMenuTipo=2. Acesso em 30 mai. 2008.

McDONALD, D. (2007) Visual Conversations Styles in Web Comunities. In: Proceedings of the $\mathbf{4 0}^{\text {th }}$ Hawaii International Conference on System Sciences. Disponível em: http://csdl2.computer.org/comp/proceedings/hicss/2007/2755/00/27550076a.pdf. Acesso em 31 mai. 2008.

MONTARDO, S. P. ; PASSERINO, L. Espelhos quebrados no ciberespaço: implicações de redes temáticas em blogs na Análise de Redes Sociais (ARS). Trabalho aceito no GT Comunicação e Cibercultura, do $17^{\circ}$. Encontro da Associação Nacional de Programas de Pós-Graduação em Comunicação (Compós). São Paulo, UNIP, 2008. Disponível em http://www.compos.org.br. Acesso em 30 mai. 2008.

MONTARDO, S.; PASSERINO, L. Estudo de blogs a partir da netnografia: possibilidades e limitações. In: RENOTE (Revista Novas Tecnologias na Educação). Vol. 4, No. 2, Dez. 2006. Disponível em: http://www.cinted.ufrgs.br/renote/dez2006/artigosrenote/25065.pdf. Acesso em 31 mai. 2008.

MOREIRA, L. M. A. ; EL-HANI, C. N. ; GUSMÃO, F. A Síndrome de Down e sua patogênese: considerações sobre o determinismo genético. In: Revista Brasileira de Psiquiatria, 2002; 22(2), pp. 9699.

PEREIRA SILVA, N. L.; DESSEN, M. A.Síndrome de Down: etiologia, caracterização e impacto na família. In: Revista Interação em Psicologia, 2002, 6 (2), p. 167-176.

PRIMO, A. Interação Mediada por Computador: A comunicação e a educação a distância segundo uma perspectiva sistêmico-relacional. Tese de Doutorado. Programa de Pós-Graduação em Informática na Educação. Universidade Federal do Rio Grande do Sul. Porto Alegre, 2003.

Interação Mútua e Interação Reativa: Uma proposta de Estudo. XXI Congresso da Intercom. Anais. Recife, PE, 1998.Disponível em <http://usr.psico.ufrgs.br/ aprimo/pb/intera.htm>. Acesso em 06 jan. 2005.

RECUERO, R. Práticas de sociabilidade em sites de redes sociais: Interação e capital social nos comentários dos fotologs. In: $17^{\circ}$. Encontro da Associação Nacional dos Programas de PósGraduação em Comunicação Social. Disponível em: http://www.compos.org.br. Acesso em 24 mai. 2008 .

Comunidades em Redes Sociais na Internet. Proposta de tipologia baseada no Fotolog.com. Tese de Doutorado. Programa de Pós-Graduação em Ciências da Comunicação e da Informação. Universidade Federal do Rio Grande do Sul. Porto Alegre, 2006.

Teoria das redes e redes sociais na Internet. Considerações sobre o Orkut, os Weblogs e os Fotologs. In: XXVII Intercom. Anais. Porto Alegre, PUCRS, 2004. Disponível em CD-Rom.

REIS, C. O. Bloody Kisses: uma aventura artística no espaço da comunicação. In: Intermídias. Disponívelemhttp://www.intermidias.com/txt/ed56/Arte_Bloody\%20kisses_Claudia\%20Connor.pdf.

Acesso em 24 mai. 2008.

RIGONI, D. A vivência dos jovens nas comunidades virtuais: indicativo para ações pedagógicas. In: Associação Brasileira de Educação à Distância. Anais. 2006. Disponível em http://www.abed.org.br/seminario2006/pdf/tc017.pdf. Acesso em 23 mai. 2008.

V. 6 № 1 , Julho, 2008 
SANZ, C. L. Imagem digital e fotologs: nova faces da temporalidade e da memória no cenário contemporâneo. In: Razón Y Palabra. México. No. 52, Ago. 2006. Disponível em: http://www.cem.itesm.mx/dacs/publicaciones/logos/anteriores/n52/26Linhares.pdf. Acesso em 24 mai. 2008.

Passageiros do tempo e a experiência fotográfica: do álbum de família ao blog digital. In: XXVIII Intercom. Anais. Rio de Janeiro, UERJ, 2005. Disponível em: Cd-Rom.

SCHWARTZMAN, J. S. Histórico. In: SCHWARTZMAN, J. S. (Org.). Síndrome de Down. São Paulo: Mackenzie, 1999a.

Generalidades. In: SCHWARTZMAN, J. S. (Org.). Síndrome de Down. São Paulo: Mackenzie, 1999b.

TELES, A. Diários virtuais/visuais: uma análise da fotografia nos fotologs. In: XXVIII Intercom. Anais. Rio de Janeiro, UERJ, 2005. Disponível em Cd-Rom. 